\title{
Biogeography: An Emerging Cornerstone for Understanding Prokaryotic Diversity, Ecology, and Evolution
}

\author{
Alban Ramette and James M. Tiedje \\ Center for Microbial Ecology, Michigan State University, 540 Plant and Soil Sciences Building, East Lansing, MI 48824-1325, USA
}

Received: 17 January 2005 / Accepted: 25 May 2005 / Online publication: 8 November 2006

\begin{abstract}
New questions about microbial ecology and diversity combined with significant improvement in the resolving power of molecular tools have helped the reemergence of the field of prokaryotic biogeography. Here, we show that biogeography may constitute a cornerstone approach to study diversity patterns at different taxonomic levels in the prokaryotic world. Fundamental processes leading to the formation of biogeographic patterns are examined in an evolutionary and ecological context. Based on different evolutionary scenarios, biogeographic patterns are thus posited to consist of dramatic range expansion or regression events that would be the results of evolutionary and ecological forces at play at the genotype level. The deterministic or random nature of those underlying processes is, however, questioned in light of recent surveys. Such scenarios led us to predict the existence of particular genes whose presence or polymorphism would be associated with cosmopolitan taxa. Furthermore, several conceptual and methodological pitfalls that could hamper future developments of the field are identified, and future approaches and new lines of investigation are suggested.
\end{abstract}

\section{Introduction}

Prokaryotic biogeography can be defined as the science that documents the spatial distribution of prokaryotic taxa in the environment at local, regional, and continental scales. In a broader sense, this discipline examines variation of microbial features (e.g., genetic, phenotypic, physiological) at different spatial scales, between distantly

Correspondence to: Alban Ramette at present address: Max Planck Institute for Marine Microbiology, Celsiusstrasse 1, 28359 Bremen, Germany; E-mail: aramette@mpi-bremen.de located sampling sites or along large environmental gradients. Its scope also encompasses the understanding of the processes generating and maintaining those distribution patterns. The ultimate goals are to propose and evaluate theories regarding the creation and evolution of such diversity patterns in the environment. Not surprisingly, this definition is identical to the one already existing for eukaryotic organisms [41], and this also may explain why current conceptual approaches share high similarities with the ones already described for eukaryotes.

The study of microbial biogeography may offer promising benefits to many research areas. Examples of application may be foreseen in the global epidemiology of human, animal, and plant pathogens; in the monitoring of the fate of inoculated biocontrol or bioremediation agents when applied at large scale; or in the identification of geographic areas where those beneficial bacteria thrive in the environment. More generally, this field will undoubtedly improve our understanding of the global microbial diversity and of the evolutionary forces shaping it.

Interest in studying spatial patterns of prokaryotes has its origin back to the beginning of the 20th century when scientists began to question the existence of different bacteria at distantly located sites $[3,5]$. Microbial biogeography has recently gained renewed interest in the microbial ecology community because of new questions about microbial diversity and the availability of powerful molecular tools to describe diversity, especially at the genotype level (e.g., [43, 48, 68]). Thus, an increasing number of studies have been published on this topic as applied to different bacterial groups in terrestrial or marine ecosystems. Biogeographic patterns associated with bacterial diversity have been reviewed elsewhere $[27,29]$.

The aims of this article were (1) to summarize the knowledge gained from studies about prokaryotic bioge- 
ography at different taxonomic levels and (2) to review the processes generating biogeographic patterns using recent evidence. Those processes are further explored on a conceptual level to tentatively draw a synthetic portrait of prokaryotic traits associated with either cosmopolitan or endemic microbes. Finally, conceptual and methodological issues that could hamper further development of this research area are discussed and future lines of investigation are suggested.

\section{What is Currently Known About Bacterial Biogeography?}

In the following sections, "species" refers to the current criteria of the bacterial species definition [64, 73], and taxon denotes any taxonomic category or group, such as a phylum, order, family, genus, or species.

Cosmopolitan Distribution. Many lines of evidence suggest that prokaryotes have a cosmopolitan distribution and in this section we give selected examples at different taxonomic levels. At the domain level, it is now well accepted that Bacteria and Archaea are globally distributed [15]. At the class level, the $\beta$-Proteobacteria, Cyanobacteria, Actinobacteria, and Flavobacteria have been shown to display worldwide distribution in marine or terrestrial ecosystems (e.g., $[4,19,58])$ ). For instance, Abell and Bowman [1] identified a large, so far uncultivated, cosmopolitan phylogenetic clade of the class Flavobacteria by sampling across a latitudinal transect in the Southern Ocean and by analyzing 16S rRNA sequence diversity. That clade was not only distributed throughout the Southern Ocean, but also widely encountered from temperate to polar seawaters, e.g., off the coast of England, the North Sea, the U.S. West Coast, and the Arctic Ocean.

At the genus level, there is a general consensus that many prokaryotes have a cosmopolitan distribution in their respective habitats [27]. For instance, the same genera of hyperthermophiles inhabit remotely located hydrothermal vent materials or deep sediments, and can survive dispersal in the open ocean in metabolically inactive states [31]. Other classical examples of cosmopolitanism among free-living bacterial genera include Polynucleobacter, which are isolated from freshwater habitats located in various climatic zones on different continents [25], fluorescent Pseudomonas and Bacillus isolated from various regions and countries $[11,59]$.

Classical examples of worldwide distribution of the same bacterial species may be found for certain human, animal, and plant pathogens. Indeed, pathogenic or commensal bacterial species, such as Escherichia coli, Haemophilus influenzae, Neisseria meningitidis, Staphylococcus aureus, and Streptococcus pneumoniae, have long been identified as cosmopolitan [45, 47], as well as certain spore-forming bacteria such as Bacillus mojavensis and B. subtilis species [59]. In plant pathology, certain species or subspecies of plant pathogens may cause huge loss in crops and orchards on a worldwide scale. Typical examples of worldwide distributions of bacterial pathogens include the case of Pseudomonas syringae causing plant canker in commercial greenhouses globally [76], Ralstonia solanacearum causing bacterial wilt on several hundreds of plant species [78], or Erwinia amylovora responsible for the fire blight disease in pears and apples on different continents [16].

At the level of 16S rRNA gene resolution, the same phylotypes have often been identified in similar habitats in different geographic areas. For instance, in the marine ecosystem, certain phylotypes of planktonic archaea were found ubiquitously in most samples investigated within eight oceanic regions [44]. In that latter study, one of the two archeal groups identified was dominant throughout the water column in the Southern Ocean and below the surface in temperate regions, whereas the other group was found dominant at the surface in temperate regions. Cosmopolitan distribution of $16 \mathrm{~S}$ rRNA sequence-based phylogenetic clusters has also been identified for freshwater bacteria using culture-independent approaches [19]. These findings are not limited to samples from water column, as high $16 \mathrm{~S}$ rRNA sequence similarities have also been evidenced between coastal and open ocean sediments (e.g., [8]). Because of the conserved nature of the molecule, evidence of cosmopolitan distribution at this level of resolution is not surprising. In fact, it is probably an inappropriate molecule to target fine biogeographic structures in environmental studies.

Endemic Taxa. Pioneering observations of noncosmopolitan distribution of prokaryotes may be found in the study of the cyanobacterial genus Synechococcus inhabiting mats in hot springs. Although certain cyanobacterial morphotypes were clearly identified in hot springs in North America, they were not observed in other hot springs around the globe, suggesting a restricted dispersal range due to physical isolation and lack of viability during transport $[10,54]$.

Although higher taxonomic levels have been identified as cosmopolitan, recent studies have clearly demonstrated that certain bacteria may not be uniformly distributed over the Earth's surface. This is contrary to a nearly century-old paradigm advocating bacterial cosmopolitanism as the rule [3], which is still generating much debate among microbial ecologists [27]. The key point, however, may be at what level the difference among cells is being evaluated, as further discussed below.

Indeed, endemic genotypes have been unequivocally identified in the soil bacteria Pseudomonas spp. [11], in the thermophilic archeon Sulfolobus "islandicus" found in water and sediments [74], or in Rhizobium legumino- 
sarum bv. phaseoli strains that live in soils and in nodules of wild and cultivated beans [70]. In those studies, target organisms were generally isolated in pure cultures and their genotypic diversity was determined based on sequence analysis of protein-coding genes [74], multilocus enzyme electrophoresis [63], or genome-wide polymerase chain reaction (PCR) amplification of repetitive elements [11]. Pairwise genetic distances between genotypes were then inferred from DNA sequences or fingerprinting patterns between isolates, and clades obtained by cluster analyses were compared with the geographic origins of the isolates. Interestingly, most of the terminal clades consisted of isolates from specific geographic locations with little or no overlap between them, indicating the existence of spatial structure in prokaryotic communities over large geographic distances, but at a relatively fine level of genetic resolution.

\section{Processes Generating Biogeographic Patterns}

Generally, the combination of speciation, extinction, and dispersal are proposed to explain the generation of biogeographic patterns. Those processes have recently been compared between eukaryotes and prokaryotes [29]. We review those processes in an evolutionary and ecological context, in order to propose theoretical scenarios leading to the formation of biogeographic patterns in prokaryotes.

Speciation. The creation and maintenance of diversity is a fundamental process in the generation and evolution of biogeographic patterns at different spatial and temporal scales. In prokaryotes, vertical speciation through descent from a common ancestor is blurred by the existence of genetic recombinations [both homologous recombination and lateral gene transfer (LGT)] [14, $20,37]$. Natural selection is thought to subsequently act on the resulting pool of diversity by sorting out genotypes better adapted to the prevailing environmental conditions through periodic selection events [13, 14]. Recombination rates have been found to be low so that they may not alter the integrity of distinct adaptations in different ecotypic lineages [14, 18]. Hence, recombination may not hinder ecologically distinct populations to diverge even further. Allopatric divergence is thus not a prerequisite for population speciation, and sympatric divergence leading ultimately to speciation could occur as soon as new adaptive traits are present in one of the members of a given ecotype. Although it is generally expected that speciation rates may be high in bacteria because of their large population sizes, the promiscuity of gene transfers, and the breadth of environmental conditions they encounter, little is know about the magnitude of those rates in nature [29].
Besides the traditional processes of prokaryotic evolution (i.e., mutation, homologous recombination, LGT, gene loss, and genome rearrangements), physical isolation has been advocated as an additional evolutionary force shaping bacterial populations [54, 67, 74, 79]. The existence of pre- and postmating barriers to gene flow could lead to the accumulation of nonselective, neutral divergences in symbiotic and free-living bacterial populations (for review, see [53]). Hence, processes such as genetic drift (random fluctuation in allele frequencies owing to sampling effects between generations), founder effects (invasion of a new environment by only few members of a taxon, which then multiply rapidly), population bottlenecks (a marked reduction of population size often resulting in a loss of genetic diversity), and neutral evolution which are commonly described in eukaryotic population genetic studies, should be given full consideration when examining prokaryotic evolution, especially in a biogeographic context. Indeed, certain examples of endemic populations were found with little correlation between the presence of specific genotypes at a given location and environmental parameters or chemical composition associated with that geographic location (e.g., [54, 74]). This may suggest that geographic isolation and subsequent neutral divergence could have had a more prominent role than adaptation to environmental conditions in those cases. However, more needs to be done on this aspect to enable more definitive conclusions to be drawn about the role of physical isolation in the generation of biogeographic patterns.

Dispersal. This is defined as the movement of populations away from their point of origin. It not only encompasses physical transport by a vector between two locations (which may be a stochastic process in the case of free-living prokaryotes), but also the establishment in, and colonization of new geographic locations by the emigrating taxa. For free-living prokaryotes, transport over large geographic distances (i.e., between continents or oceans) may be possible through the combination of favorable climatic factors (e.g., winds, storms), disseminating vectors (e.g., oceanic currents, dust, plant seeds, surfaces of humans, birds, or insects migrating between regions), and sometimes the existence of survival or dormant stages in bacteria that would enhance their chance to endure long-distance transport (for review, see $[22,29])$. Although it is thought that microbial transport may be an ongoing process [22], it is not yet known how many of those transported microorganisms may survive their journey and subsequently establish into new environments. Scarce data are available concerning the dispersal of free-living bacteria in the environment because molecular techniques have not reached the level of resolution that would enable an easy track- 
ing of a given genotype in space and time (sampling issues are further discussed below).

The distribution patterns of symbionts, pathogens, or commensals may mostly be dictated by their hosts' specific biogeography. This is the case, for instance, for human pathogens that are increasingly spread worldwide due to human activity [17, 21, 47]. In an evolutionary context, dispersal may also be seen as a stochastic process enabling taxa to physically escape the purging of diversity caused by periodic selection that potentially may lead members of a same ecotypic lineage to extinction [53].

To quantify the extent of migration between spatially separated populations, migration rates have generally been inferred from molecular data (e.g., protein-coding gene sequences). In the case of two Bacillus species, migration rates were found to be the highest between close locations, but still prevented genetic drift to occur even among the most distant populations [59]. Therefore, genetic divergence was found to be limited between local and more distant populations, i.e., those populations displayed a cosmopolitan distribution. Similarly, for Bradyrhizobium canariense and B. japonicum, two sympatric species sharing partially the same ecological niches (i.e., the same root nodules), recombination and migration may represent significant evolutionary forces that provide internal cohesiveness, shape their population genetic structure, and may prevent their geographical differentiation [74].

Barriers to dispersal, i.e., areas where taxa cannot establish because of physical barriers (e.g., topography), physiological requirements (e.g., narrow temperature, salinity, or $\mathrm{pH}$ tolerance range), ecological constraints (e.g., higher competition), or because of limited resource availability between favorable patches, have been proposed to explain the existence of endemic patterns (for review, see [53]). For instance, different Flavobacteria communities seem to be selected based on both the physical barrier of the polar front and water temperature [1]. For thermophilic Sulfolobus inhabiting geothermal hot springs, Whitaker et al. [74] showed that subpopulations were spatially isolated from each other, probably as the result of their specific physiological requirements (i.e., low $\mathrm{pH}$, high temperature) that would reduce their chance to colonize inhospitable habitats between geothermal regions.

Extinction. It is generally postulated that extinction may be rare in prokaryotes because of their large population sizes, high growth rates, and/or the presence of survival or dormant states when facing harsh environmental conditions [29]. However, little data exist in the literature about how common such characteristics may be in natural populations. Such generalizations therefore remain very questionable.
Protection against adverse environmental conditions has yet been observed in Pseudomonas aeruginosa that may undergo extensive self-generated diversification within its biofilms [6]. Interestingly, in that process, induced pleiotropic effects could lead to the appearance of genotypes with increased dissemination abilities and greater resistance to environmental stresses. Such biological "insurance" may therefore contribute significantly to the reduction of the risk of extinction, especially when colonies are transported further away from their original niches or when local resources have been depleted. However, the existence and magnitude of this mechanism in other prokaryotes is not yet known.

As for the speciation and dispersal processes, extinction rates may be difficult to measure in situ and indirect methods based on inference from genetic data have been proposed to estimate those parameters [49]. Thus, using simple genealogical models, theoretical studies have shown that diversity could be concentrated in small subpopulations, whose extinction could cause fluctuations of diversity even in the absence of extrinsic perturbations [57]. Those results were found to agree with biogeographic data obtained for Pseudomonas spp. populations [11, 57], and strengthened the idea that extinction may dramatically impact prokaryotic diversity.

Although speciation may initially occur at a small scale and dispersal is supposed to happen at a larger scale, the spatial scale relative to extinction may be more difficult to grasp and may depend on the joint effects of dispersal and speciation. Moreover, it is not known to what extent extinction is either stochastic or deterministic. Indeed, abiotic and biotic factors leading to prokaryotic population survival or extinction in the environment are still poorly understood.

\section{Biogeographic Patterns in an Evolutionary Con-} text. Based on the current prokaryotic evolutionary theory, we examine in this section two extreme cases, starting from parental lineages that are either cosmopolitan or endemic, and then discuss possible outcomes in an evolutionary context.

A cosmopolitan taxon may intrinsically be particularly suited to succeed in contrasting environmental conditions owing to, for instance, a high genome plasticity and metabolic versatility as may be the case for the previously mentioned Pseudomonas genus of the Proteobacteria. Such taxa would display a cosmopolitan distribution as long as dispersal occurs over large geographic distances in a continuous manner (see "Dispersal") and genetic divergence is limited in the same time frame. However, ecological divergence and geographic isolation may ultimately take place over time and would not be counterbalanced by recombination [13], leading local populations to be more genetically distinct from 
the ancestral cosmopolitan population. As aforementioned, transport of free-living prokaryotes may be a stochastic process, which implies that a part of local populations would not disperse and would be more subject to genetic divergence. If adaptive mutants appear within the resident population and enough time is given for periodic selection to occur, they would purge the diversity locally, because they may be better adapted than their congeners for a given set of local, environmental conditions. Additionally, newly immigrating cosmopolitan ecotypes would also be outcompeted by those adaptive mutants, leading to even more differentiation of the locally vs. globally distributed populations.

It may, however, be argued that dispersal may favor the replacement of those latter adaptive mutants by other adaptive mutants coming from other locations, which would have arisen in parallel and could potentially harbor even better adaptations for the given set of local conditions. Although it is difficult to predict the outcome of those interactions in such a theoretical approach, the ultimate results would probably be that cosmopolitan taxa would tend to be more endemic, given enough evolutionary time to pass. If this scenario holds true, we may be able (1) to find genetic markers (gene presence, absence, or polymorphism) indicating the cosmopolitan nature of those organisms, and (2) to significantly correlate genetic modifications of those markers with a change in the ability of those organisms to be globally distributed.

In the case of endemic taxa, ecological and neutral divergence would lead to even more patterns of endemism, leading potentially to extinction if the environmental conditions are met only locally and dissemination is limited. However, LGT events that may be rare but promiscuous have a significant impact on the ecology and speciation of prokaryotes [14]. Acquisition of new ecological traits may offer new possibilities to the organism, such as the invasion of new niches, and colonization of new habitats, which could distantly be located from the original site of endemism. Noticeably, the chance to acquire those putative genes associated with cosmopolitan traits may be high, as those genes would logically be more frequent in nature than endemism-related ones (because cosmopolitan taxa are everywhere, they are more likely to be donors of genetic information). The final outcome would be a gradual expansion of the habitat or niche range of such taxa, ultimately leading to a cosmopolitan distribution. In this view, cosmopolitanism is clearly posited to be selected for by natural selection. However, it is not known to what extent traits associated with global distributions are selected for in natural populations. Noteworthy, the acquisition of new traits may also occur in cosmopolitan taxa, which would potentially counterbalance their unavoidable differentiation as a result of geographic isolation and ecological divergence.

Interestingly, the second scenario consisting of a range expansion from endemic to cosmopolitan taxa may be supported by observations of the well-documented evolution of human pathogens. Acquisition of new virulence factors or intragenomic recombinations have been proposed to explain recent, global dispersal of clonal lineages (clonal expansion), which were formerly known to occur only locally (e.g., [34, 47, 62]). This illustrates that evolution in prokaryotes may lead to dramatic changes in their biogeographic patterns, because prokaryotic biology and ecology are both affected during the evolutionary process.

How long could biogeographic patterns be stable? Examples from bacterial pathogens may again offer first glimpses of the longevity of clonal lineages, although they might not be good representatives for the whole prokaryotic diversity. Depending on the level of clonality of a given species, longevity of clonal lineages have been suggested to last from few decades for rapidly diversifying and recombining $N$. meningitis clones, to thousands of years for the more clonal Salmonella enterica, to more than 35,000 years for Shigella clones [36]. Therefore, it seems that future biogeographic studies would have to take into account the variation of evolutionary rates in different lineages to better understand prokaryotic distribution patterns and their likely evolution at different spatial and temporal scales.

Summarizing, evolution in prokaryotes may potentially have two contrasting effects: either the organisms may become even more specialized to their niches and tend to be restricted to the same area, or they may acquire new ecological traits that would make them more prone to invade new niches or habitats and disperse. Although cosmopolitan and endemic distributions may be two extreme, opposite distributions, many prokaryotic taxa would probably have a distribution in between those two extremes, by being, for instance, present as patches distributed in many regions without yet displaying a cosmopolitan distribution (Ramette and Tiedje, unpublished data). This could reflect the fact that the formation of biogeographic patterns may be a dynamic process of expansion or regression of the habitat range at the genotype level, which may be governed by the complex interplay of the nature of the genes gained, lost, or modified, the extent of dispersal and extinction, and natural selection.

\section{Conceptual and Methodological Limitations}

The study of microbial biogeography must cope with several conceptual and methodological limitations, which dramatically influence each other. Approaches chosen by environmental microbiologists are intimately related to 
their a priori conceptions about biogeography and microbial behavior at larger scales, which were mostly inherited from biogeographic studies of eukaryotic organisms.

Many of the practical issues discussed in this section are not only specific to prokaryotic biogeography, but are common to environmental microbial ecology in general. The fact that large spatial and potentially temporal scales are involved in biogeographic approaches may yet add another layer of complexity to experimental design and data analyses.

Theoretical Foundations. Because spatial and temporal scales are often too large for experimentation to be carried out, theories of biogeography are generally developed by searching for patterns, formulating theories, and testing their assumptions and predictions for a limited number of data. Such predictions may include taxa presence/absence, their interactions, their relative abundance, and their persistence in time. For prokaryotes, the finding of such biodiversity rules would be a major advance in the ability to predict the evolution of diversity patterns of natural populations in the environment, which is so far poorly understood.

It may be fruitful to question whether biogeographic theories that have been developed and refined for plants and animals would also fit for prokaryotes. The "island" model of MacArthur and Wilson [42] certainly constitutes a starting point to address that question. Indeed, recent studies (e.g., $[54,74]$ ) showing that endemism was more common than previously thought used the "island" model that consisted of well-isolated habitats (e.g., hot springs) naturally occurring in geographically distant areas. The rationale was that, from an extremophile's perspective, extreme environments may be seen as island-like habitats dispersed in an inhospitable environment, leading to geographic isolation and potentially to subsequent neutral divergence. Although the island-like nature of hot springs was essential to reveal endemic patterns, predictions based on island biogeographic theories have yet not been extensively tested using those prokaryotic diversity data. Indeed, the "mainland" that represents the source area of taxa that emigrate to those islands may be difficult to define, thus limiting inferences about rates of dispersal and extinction, which would be necessary to assess the validity of such theoretical predictions in the prokaryotic case.

The niche-based equilibrium view is, so far, dominant among microbiologists and constitutes the foundation of the actual evolutionary theory for prokaryotes (e.g., [14]), which aims to explain ecological divergence and ultimately speciation. Neutral processes of diversification, such as genetic drift mediated by geographic isolation, have challenged this unique view and were suggested as additional evolutionary factors to be con- sidered in prokaryotic evolution [53]. It is thus relevant to quantify the relative importance of those different evolutionary forces, and to determine whether they could be better represented by either niche-based (i.e., taxon specific) or neutral (i.e., ecological equivalence of taxa, sensu Hubbell [30]) models of diversification leading to the generation of biogeographic patterns in prokaryotes. A reconciliation of those two perspectives, which should not be mutually exclusive, would also be much needed to produce a unified theory.

Although an extensive review of the existing biogeography and biodiversity theories goes beyond the scope of the present review, future lines of exploration would benefit from comparing prokaryotic biodiversity data with theoretical predictions coming from, e.g., the unified neutral theory of biodiversity and biogeography [30], and metacommunity paradigms such as the speciessorting, the mass-effect, or the patch-dynamic models (for review about their scopes, assumptions, and predictions, see, e.g., [39]). The comparison of observed vs. theoretical diversity patterns would pave the way toward developing future biodiversity and biogeography theories for prokaryotes, whose ultimate goals would be to link evolutionary and ecological perspectives into a common framework.

Species Concept and Taxonomic Level of the Study. As probably influenced by plant and animal biogeographic approaches, the species is also the most commonly found unit in microbial biogeographic studies. However, the meaning of the current bacterial species definition is still much debated $[35,60]$, because it does not take into account the evolutionary forces that provide cohesiveness to bacterial population structure $[14,71,74]$. Although a polyphasic approach combining both phenotypic and genotypic characters has been recommended to help classify new strains into species $[64,69]$, species are sometimes identified as operational taxonomic units based on the similarity of 16S rRNA gene sequences for practical reasons in environmental studies. The reliance on that unique ribosomal gene to assess similarity in microbial biogeography may lead to premature conclusions about the biogeographic distribution of the target taxa due to a lack of resolving power, and hence may lead to erroneous conclusions about the ecological significance of bacterial diversity [32].

It may however be argued that if biogeographic patterns can already be observed using 16S rRNA genebased approaches, this may indicate even stronger spatial structure of the populations when molecular tools with finer levels of resolution are used (this rationale was used in, e.g., $[28,54])$. Besides, $16 \mathrm{~S}$ rRNA-based approaches may still be valuable as the first steps into identifying and 
further describing biogeographic patterns for uncultured taxa in the environment. Indeed, few data have yet been obtained for the vast unseen majority of uncultured microorganisms that represent more than $99 \%$ of prokaryotic life in seawater, sediments, and soil [2].

Choice of the Target Taxa. It is well established that microbial diversity is unparalleled in the biological world and a large number of prokaryotic taxa present in the environment are still to be discovered [2, 66, 72]. However, most of the recent biogeographic studies dealt with only culturable species, and it may be questionable to extrapolate results based on those few selected taxa to prokaryotic biogeography in general. The choice of target organisms is often dictated by practical issues such as natural abundance in the environment or ease of isolation and storability, as may be the case for Bacillus or Pseudomonas, as previously mentioned.

Different taxa may respond differently to evolutionary and ecological processes governing the creation and maintenance of biogeographic patterns at different scales. One challenge of microbial biogeography is therefore to identify the magnitude of those underlying principles and to determine at what taxonomic levels those forces operate. In that process, it may be important to identify whether "indicator" taxa exist and to what extent their distribution patterns could be representative of those of other taxa.

Methodological Issues. The increasing number of tools to resolve microbial microdiversity has provided access to more genetic information about environmental bacterial populations and communities, which in turn has significantly helped the reemergence of the study of prokaryotic biogeography. However, several pitfalls in the methodological approaches have to be recognized and, if possible, avoided in future studies. Issues relating to the study of microbial ecology in general may be dramatically amplified when dealing with biogeographic approaches, because the latter try to draw conclusions over large spatial scales based on a limited number of samples. In the following sections, we review concerns about the sampling process in a spatial and temporal context, and discuss the central issue of resolution and saturation of molecular tools used.

Sampling Issue and Spatial Scale. Conversely to plant and animal biogeographic studies, bacterial biogeography suffers from the destructive nature of the sampling process that hinders rigorous monitoring of the evolution of diversity at the same locations. It is thus not surprising that, although clear endemic, patchy, or cosmopolitan patterns have been evidenced in several prokaryotic taxa, scant data exist on the stability of those patterns in time or about their seasonal fluctuations.

Another critical issue is the size of environmental samples from which diversity data are derived. For instance, it is technically and practically difficult to scale up sample size from $1 \mathrm{~g}$ to $1 \mathrm{~kg}$ of soil or sediment. However, assessment of biogeographic patterns shifts with various spatial scales is essential not only for sampling reasons but also for a better understanding of the ecological principles at play. Such an approach was followed by, for instance, Nicol and colleagues [50], who examined the heterogeneity of archaeal community structure in grassland soil at two contrasting spatial scales using $16 \mathrm{~S}$ rRNA-based analyses. At a macroscale, single core samples were found to be mostly representative of the heterogeneity across larger transects (i.e., for soil cores distant of 2-m intervals). However, microscale analyses revealed that community structure varied significantly within single cores, and more similarity was evidenced for samples consisting of $10 \mathrm{~g}$ soil than for smaller sample sizes (i.e., 1 and $0.1 \mathrm{~g}$ ). Interestingly, similar results were found for cold marine sediments where community shifts were generally evidenced between different sediment layers, whereas geographical differences appeared to result in smaller differences [7]. Thus, one essential methodological step would be to determine the validity and implications of using rather small sample sizes to describe prokaryotic ecology and diversity at larger spatial scales.

Because biogeographic pattern analyses are initially a mere description of taxa presence or absence in different samples, one way to ascertain the absence of target organisms may be to perform a posteriori controls of all target-negative samples. For instance, lineage-specific PCR (e.g., [54]), combined or not with enrichment steps when population abundance in the samples is thought to be low (e.g., [56]), may be used to validate the presence (or absence) of rare genotypes. Alternatively, direct microscopic observations of the samples (e.g., using fluorescent in situ hybridization with taxon-specific probes) or the utilization of various physiological methods to identify prokaryotic activity (e.g., $[2,46,65])$ may be used.

The third dimension of the habitat being sampled, which can be either soil, sediment layers, or water columns, may constitute another challenge. Hitherto, microbial biogeographic studies have mostly focused on the Earth's surface, probably in a similar way as being done for plants and animals. Nevertheless, few studies dealing with large scales and depth may be found in the marine environment or sediments (e.g., $[4,8]$ ). Such studies illustrate that still much is to be learned from vertical analysis of the diversity of our planet, all the more as prokaryotic life has been evidenced kilometers below the Earth's surface [75]. 
Although certain studies focus on microbial biogeography between sites, regions, and continents, another line of research has also been followed, which consists of examining microscale patterns of bacterial populations [23]. The rationale behind this approach is that synecological and autecological interactions between the target organisms and their surrounding environment occur in microenvironments, at a microscale [24]. The microscale and macroscale approaches that aim at better understanding spatial diversity patterns should be integrated into a common framework. Indeed, different ecological and evolutionary principles may occur at different scales. As outlined below, new analytical tools need to be explored or developed to combine such data into a coherent perspective.

Resolution and Saturation of the Methods. Molecular methods with increasing power of resolution were used to demonstrate that the choice of molecular tools has a profound impact on our final interpretation of biogeographic patterns [11]. In that study, when methods with a coarse level of resolution such as 16S rDNA and ITS restriction analyses were used, fluorescent pseudomonas populations displayed a cosmopolitan and weakly endemic distribution, respectively, whereas the use of whole-genome fingerprinting methods (i.e., rep-PCR [55]) revealed that nearly all fluorescent Pseudomonas genotypes were endemic to one geographic area, with little overlap between regions and sites. Therefore, using molecular tools with weak resolving power could hide the existence of small communities with their particular biogeographic patterns. A combination of methods of increasing resolving power may be a good approach to study biogeography in prokaryotes, because they would offer the advantage of looking at the same organisms with different perspectives, and would target different levels of similarity.

Genetic relationships inferred from whole-genome typing (e.g., rep-PCR) approach saturation when similarity values are near or under $65 \%[11,55,70]$. This indicates that it may not be possible to use such techniques to confidently compare higher taxonomic levels, and that alternate methods are therefore needed. For each methodology, the levels of saturation and resolution have to be experimentally determined to better understand the biases and limits of the chosen approach. In that respect, fingerprinting methods such as rep-PCR may be initially used to sort out clonemates, and then more targeted approaches such as sequence analysis of multiple protein-coding genes may be used to analyze phylogenetic, biogeographic, and evolutionary patterns. For instance, Whitaker and colleagues [74] showed that the concatenation of the sequences of nine proteincoding genes was necessary to obtain enough resolution to indicate endemic clades, whereas the analysis of those gene sequences taken individually could not provide that level of resolution. Although rep-PCR and multilocus sequence analyses provide precious tools to type genotypes into clonal lineages, there is still no comparable tool to assess the genotypic diversity for uncultured prokaryotes. New methodological developments are therefore needed to explore this field.

\section{Future Developments}

Despite the existence of numerous pitfalls when dealing with prokaryotic biogeography, new developments at the conceptual and methodological levels would dramatically improve our understanding of spatial and temporal patterns of microbial life in different habitats. Here, a series of potential developments is thus proposed to stimulate future research effort.

Experimental Evaluation of Biogeography Theory for Prokaryotes. Because spatial and temporal scales are too large for mechanistic experiments to be performed in situ, testing biogeographic hypotheses would remain a formidable task. However, working with prokaryotes has tremendous advantages because of their small size, short generation time, and the huge availability of molecular tools and, generally, of biological and physiological information. Thus, transposition of complex ecological questions to simpler laboratory-based model systems would be extremely valuable (for review, see [33]) in future biogeographic studies. The main objectives of such approaches would be to deconstruct and simplify the effects of spatial and temporal scales on complex systems into individual parts in order to study them with high degree of experimental control and reproducibility (e.g., [67]).

Taxonomic Level, Representative Taxa, and Sampling. As mentioned above, a current question in prokaryotic biogeography is to understand at which genetic level (or taxonomic level) significant geographic differences can be observed. This approach would provide first bases for a more synthetic and predictive view of prokaryotic biogeography and its ecological meaning. Although studies based on single species may still be needed to obtain more field data on which theories and hypotheses can be built, efforts should also be directed toward describing multiple coexisting species (comparative biogeography), so that a more general picture can emerge.

A promising approach would be to systematically compare samples from various natural habitats to draw an overview about genetically coherent and incoherent areas and their scale dependency. This would help determine regions or sites of high and low diversity to be further investigated and would provide clues about 
the interplay of major biotic and abiotic parameters that would lead to such situations. Areas of higher endemism would then be the necessary targets of conservation biology programs.

Beyond the mere description of diversity patterns, future research should also focus on the spatial and temporal distribution of the resulting ecological functions and properties. The biogeography of microbial functions would be a specificity of the prokaryotic model compared with that of plants and animals. It is therefore essential for microbial ecologists to undertake this line of research.

Technical Developments. The reemergence of the study of microbial biogeography was made possible by the spectacular technical breakthroughs to resolve microbial diversity at the genotype level. It may therefore be hypothesize that the future availability of methods with higher resolving power would indubitably be central in providing new concepts in prokaryotic biogeography. In that context, microarray technology and genomics (for review, see for instance [12]) may thus offer promising tools to obtain fine-scale description of the genetic background of organisms in various habitats [51]. For instance, a novel type of microarray consisting of whole genomic DNA isolated from 67 representative bacterial strains has been designed to identify and detect specifically, sensitively, and quantitatively microorganisms in environmental samples [77]. However, to our knowledge, no such applications have yet been carried out to document biogeographic distribution of prokaryotes.

Hitherto, sequencing of protein-coding genes has been shown to be an efficient method to analyze microbial biogeographic data of culturable prokaryotes (e.g., [74]). However, such approach traditionally relies on analyzing a given set of conserved genes [61]. The fact that bacterial genomes could be split into a core of housekeeping genes and accessory genes that encode for adaptive traits $[20,36,52]$ offers great promise for the study of the evolution and phylogeny of those accessory genes in concord with biogeographic patterns. This could potentially shed light on a set of genes or single nucleotide polymorphisms (SNP) that would generally be associated with cosmopolitan or endemic organisms.

Development or Utilization of New Analytical Tools. Microbial biogeographic studies are per se multidisciplinary because of the breadth of the concepts and tools used. They require knowledge in many fields such as (microbial) ecology, (geo)statistics, mathematics, geology, phylogenetics, and population genetics. Therefore, future challenges may rely on how to constructively integrate those different fields of expertise to provide beneficial and coherent grounds for the development of a microbial biogeography theory.
As phylogenetic tools may be central to the understanding of modern environmental microbiology, more effort should be put toward integrating spatial statistical tools in the microbial ecologist toolbox. Introductory reviews illustrating the integration of spatial scale in ecology may be found in, for instance, Liebhold and Gurevitch [40] or in the classical reference book of Legendre and Legendre [38].

Other scientific fields should also be explored to develop new analytical and conceptual tools that would systematically include spatial scales in environmental microbiology studies. In that context, more interactions between microbial ecologists, mathematicians, and statisticians should necessarily be fostered to address issues specific to the prokaryotic case. It would be interesting, for instance, to determine whether the generation of biogeographic patterns in prokaryotes is either deterministic or random, or neither of the two (i.e., chaotic). Advances in other disciplines such as the science of complexity [9, 26] may thus offer new lines of investigation.

\section{Acknowledgments}

We thank J. Fredrickson and Y. Cohen for the invitation to write this article and two anonymous reviewers for helpful comments.

We acknowledge support from NSF grant no. DEB00755564, DOE Office of Science (BER) DE-FG0298ER62535, and a postdoctoral fellowship from the Swiss NSF to A.R.

\section{References}

1. Abell, GCJ, Bowman, JP (2005) Ecological and biogeographic relationships of class Flavobacteria in the Southern Ocean. FEMS Microbiol Ecol 51: 265-277

2. Amann, RI, Ludwig, W, Schleifer, KH, Torsvik, VL, Goksoyr, J (1995) Phylogenetic identification and in situ detection of individual microbial-cells without cultivation. Microbiol Rev 59: 143-169

3. Baas-Becking, LGM (1934) Geobiologie of Inleiding Tot de Milieukunde. Van Stockkum \& Zoon, The Hague, The Netherlands

4. Bano, N, Ruffin, S, Ransom, B, Hollibaugh, JT (2004) Phylogenetic composition of Arctic Ocean archaeal assemblages and comparison with Antarctic assemblages. Appl Environ Microbiol 70: 781789

5. Beijerinck, MW (1913) De infusies en de ontdekking der backteriën, Jaarboek van de Koninklijke Akademie v. Wetenschoppen. Muller. Amsterdam, The Netherlands

6. Boles, BR, Thoendel, M, Singh, PK (2004) Self-generated diversity produces 'insurance effects' in biofilm communities. Proc Natl Acad Sci USA 101: 16630-16635

7. Bowman, JP (2004) Psychrophilic prokaryote structural-functional relationships, biogeography and evolution within marine sediment. Cell Mol Biol 50: 503-515

8. Bowman, JP, McCuaig, RD (2003) Biodiversity, community structural shifts, and biogeography of prokaryotes within Antarctic continental shelf sediment. Appl Environ Microbiol 69: 2463-2483 
9. Brown, JH, Gupta, VK, Li, BL, Milne, BT, Restrepo, C, West, GB (2002) The fractal nature of nature: power laws, ecological complexity and biodiversity. Philos Trans R Soc Lond, B 357: 619-626

10. Castenholz, RW (1978) The biogeography of hot spring algae through enrichment cultures. Mitt Int Ver Limnol 21: 296-315

11. Cho, JC, Tiedje, JM (2000) Biogeography and degree of endemism of fluorescent Pseudomonas strains in soil. Appl Environ Microbiol 66: $5448-5456$

12. Coenye, T, Gevers, D, dePeer, YV, Vandamme, P, Swings, J (2005) Towards a prokaryotic genomic taxonomy. FEMS Microbiol Rev 29: $147-167$

13. Cohan, FM (2004) Concepts of bacterial biodiversity for the age of genomics. In: Fraser, CM, Read, TD, Nelson, KE (Eds.) Microbial Genomes. Humana Press, Totowa, NJ, pp 175-194

14. Cohan, FM (2002) What are bacterial species? Annu Rev Microbiol 56: $457-487$

15. DeLong, EE, Pace, NR (2001) Environmental diversity of Bacteria and Archaea. Syst Biol 50: 470-478

16. Eastgate, JA (2000) Erwinia amylovora: the molecular basis of fireblight disease. Mol Plant Pathol 1: 325-329

17. Falush, D, Wirth, T, Linz, B, Pritchard, JK, Stephens, M, Kidd, M, Blaser, MJ, Graham, DY, Vacher, S, Perez-Perez, GI, Yamaoka, Y, Megraud, F, Otto, K, Reichard, U, Katzowitsch, E, Wang, XY, Achtman, M, Suerbaum, S (2003) Traces of human migrations in Helicobacter pylori populations. Science 299: 1582-1585

18. Feil, EJ, Spratt, BG (2001) Recombination and the population structures of bacterial pathogens. Annu Rev Microbiol 55: 561-590

19. Glöckner, FO, Zaichikov, E, Belkova, N, Denissova, L, Pernthaler, J, Pernthaler, A, Amann, R (2000) Comparative 16S rRNA analysis of lake bacterioplankton reveals globally distributed phylogenetic clusters including an abundant group of actinobacteria. Appl Environ Microbiol 66: 5053-5065

20. Gogarten, JP, Doolittle, WF, Lawrence, JG (2002) Prokaryotic evolution in light of gene transfer. Mol Biol Evol 19: 2226-2238

21. Gordon, DM (2001) Geographical structure and host specificity in bacteria and implications for tracing the source of coliform contamination. Microbiology 147: 1079-1085

22. Griffin, DW, Kellogg, CA, Garrison, VH, Shinn, EA (2002) The global transport of dust-an intercontinental river of dust, microorganisms and toxic chemicals flows through the Earth's atmosphere. Am Sci 90: 228-235

23. Grundmann, GL (2004) Spatial scales of soil bacterial diversity-the size of a clone. FEMS Microbiol Ecol 48: 119-127

24. Grundmann, GL, Debouzie, D (2000) Geostatistical analysis of the distribution of $\mathrm{NH}_{4}$ and $\mathrm{NO}_{2}$ oxidizing bacteria and serotypes at the millimeter scale along a soil transect. FEMS Microbiol Ecol 34: $57-62$

25. Hahn, MW (2003) Isolation of strains belonging to the cosmopolitan Polynucleobacter necessarius cluster from freshwater habitats located in three climatic zones. Appl Environ Microbiol 69: 5248-5254

26. Halley, JM, Hartley, S, Kallimanis, AS, Kunin, WE, Lennon, JJ, Sgardelis, SP (2004) Uses and abuses of fractal methodology in ecology. Ecol Lett 7: 254-271

27. Hedlund, BP, Staley, JT (2003) Microbial endemism and biogeography. In: Bull, AT (Ed.) Microbial Diversity and Bioprospecting. ASM Press, Washington, DC, pp 225-231

28. Horner-Devine, MC, Lage, M, Hughes, JB, Bohannan, BJM (2004) A taxa-area relationship for bacteria. Nature 432: 750-753

29. Horner-Devine, MC, Carney, KM, Bohannan, BJM (2003) An ecological perspective on bacterial biodiversity. Proc R Soc Lond, B 271: $113-122$

30. Hubbell, SP (2001) The Unified Neutral Theory of Biodiversity and Biogeography. Princeton Monographs in Population Biology. Princeton University Press, Princeton, NJ, 448 pp
31. Huber, R, Stoffers, P, Cheminee, JL, Richnow, HH, Stetter, KO (1990) Hyperthermophilic archaebacteria within the crater and open-sea plume of erupting Macdonald Seamount. Nature 345: 179-181

32. Jaspers, E, Overmann, J (2004) Ecological significance of microdiversity: identical $16 \mathrm{~S}$ rRNA gene sequences can be found in bacteria with highly divergent genomes and ecophysiologies. Appl Environ Microbiol 70: 4831-4839

33. Jessup, CM, Kassen, R, Forde, SE, Kerr, B, Buckling, A, Rainey, PB, Bohannan, BJM (2004) Big questions, small worlds: microbial model systems in ecology. Trends Ecol Evol 19: 189-197

34. Johansson, A, Farlow, J, Larsson, P, Dukerich, M, Chambers, E, Bystrom, M, Fox, J, Chu, M, Forsman, M, Sjostedt, A, Keim, P (2004) Worldwide genetic relationships among Francisella tularensis isolates determined by Multiple-Locus Variable-Number Tandem Repeat analysis. J Bacteriol 186: 5808-5818

35. Konstantinidis, KT, Tiedje, JM (2005) Genomic insights that advance the species definition for prokaryotes. Proc Natl Acad Sci USA 102: 2567-2572

36. Lan, R, Reeves, PR (2001) When does a clone deserve a name? A perspective on bacterial species based on population genetics. Trends Microbiol 9: 419-424

37. Lawrence, JG (2002) Gene transfer in bacteria: speciation without species? Theor Popul Biol 61: 449-460

38. Legendre, P, Legendre, L (1998) Numerical Ecology, 2nd English edn, Elsevier, Amsterdam

39. Leibold, MA, Holyoak, M, Mouquet, N, Amarasekare, P, Chase, MF, Hoopes, MF, Holt, RD, Shurin, JB, Law, R, Tilman, D, Loreau, M, Gonzalez, A (2004) The metacommunity concept: a framework for multi-scale community ecology. Ecol Lett 7: 601613

40. Liebhold, AM, Gurevitch, J (2002) Integrating the statistical analysis of spatial data in ecology. Ecography 25: 553-557

41. Lomolino, MV (2000) A call for a new paradigm of island biogeography. Glob Ecol Biogeogr 9: 1-6

42. MacArthur, RH, Wilson, EO (1967) The Theory of Island Biogeography. Princeton University Press, Princeton, NJ

43. Maiden, MCJ, Bygraves, JA, Feil, E, Morelli, G, Russell, JE, Urwin, R, Zhang, Q, Zhou, JJ, Zurth, K, Caugant, DA, Feavers, IM, Achtman, M, Spratt, BG (1998) Multilocus sequence typing: a portable approach to the identification of clones within populations of pathogenic microorganisms. Proc Natl Acad Sci USA 95: 3140-3145

44. Massana, R, DeLong, EF, Pedros-Alio, C (2000) A few cosmopolitan phylotypes dominate planktonic archaeal assemblages in widely different oceanic provinces. Appl Environ Microbiol 66: 1777-1787

45. Maynard Smith, J, Dowson, CG, Spratt, BG (1991) Localized sex in bacteria. Nature 349: 29-31

46. Molin, S, Givskov, M (1999) Application of molecular tools for in situ monitoring of bacterial activity. Environ Microbiol 1: 383-391

47. Musser, JM (1996) Molecular population genetic analysis of emerged bacterial pathogens: selected insights. Emerg Infect Dis 2: $1-17$

48. Muyzer, G (1998) Structure, function and dynamics of microbial communities: the molecular biological approach. In: Carvalho, GR (Ed.) Advances in Molecular Ecology. IOS Press, Amsterdam, pp 87-117

49. Nee, S, Holmes, EC, May, RM, Harvey, PH (1994) Extinction rates can be estimated from molecular phylogenies. Philos Trans R Soc Lond, B 344: 77-82

50. Nicol, GW, Glover, LA, Prosser, JI (2003) Spatial analysis of archaeal community structure in grassland soil. Appl Environ Microbiol 69: 7420-7429

51. Ochman, H, Santos, SR (2005) Exploring microbial microevolution with microarrays. Infect Genet Evol 5: 103-108 
52. Ochman, H, Lawrence, JG, Groisman, EA (2000) Lateral gene transfer and the nature of bacterial innovation. Nature 405: 299-304

53. Papke, RT, Ward, DM (2004) The importance of physical isolation to microbial diversification. FEMS Microbiol Ecol 48: 293-303

54. Papke, RT, Ramsing, NB, Bateson, MM, Ward, DM (2003) Geographical isolation in hot spring cyanobacteria. Environ Microbiol 5: 650-659

55. Rademaker, JLW, Louws, FJ, De Bruijn, FJ (1998) Characterization of the diversity of ecologically important microbes by rep-PCR genomic fingerprinting. Mol Microb Ecol Man. 3.4.3: 1-27

56. Ramette, A, LiPuma, JJ, Tiedje, JM (2005) Species abundance and diversity of Burkholderia cepacia complex in the environment. Appl Environ Microbiol 71: 1193-1201

57. Rauch, EM, Bar-Yam, Y (2004) Theory predicts the uneven distribution of genetic diversity within species. Nature 431: 449-452

58. Rejmánková, E, Komárek, J, Komárková, J (2004) Cyanobacteria—a neglected component of biodiversity: patterns of species diversity in inland marshes of northern Belize (Central America). Divers Distrib 10: 189-199

59. Roberts, MS, Cohan, FM (1995) Recombination and migration rates in natural populations of Bacillus subtilis and Bacillus mojavensis. Evolution 49: 1081-1094

60. Rosselló-Mora, R, Amann, R (2001) The species concept for prokaryotes. FEMS Microbiol Rev 25: 39-67

61. Santos, SR, Ochman, H (2004) Identification and phylogenetic sorting of bacterial lineages with universally conserved genes and proteins. Environ Microbiol 6: 754-759

62. Schouls, LM, van der Heide, HGJ, Vauterin, L, Vauterin, P, Mooi, FR (2004) Multiple-Locus Variable-Number Tandem Repeat analysis of Dutch Bordetella pertussis strains reveals rapid genetic changes with clonal expansion during the late 1990s. J Bacteriol 186: 5496-5505

63. Souza, V, Nguyen, TT, Hudson, RR, Pinero, D, Lenski, RE (1992) Hierarchical analysis of linkage disequilibrium in rhizobium populations: evidence for sex. Proc Natl Acad Sci USA 89: 8389-8393

64. Stackebrandt, E, Frederiksen, W, Garrity, GM, Grimont, PAD, Kämpfer, P, Maiden, MCJ, Nesme, X, Rosselló-Mora, R, Swings, J, Trüper, HG, Vauterin, L, Ward, AC, Whitman, WB (2002) Report of the ad hoc committee for the re-evaluation of the species definition in bacteriology. Int J Syst Evol Microbiol 52: 1043-1047

65. Staley, JT, Gosink, JJ (1999) Poles apart: biodiversity and biogeography of sea ice bacteria. Annu Rev Microbiol 53: 189-215

66. Torsvik, V, Ovreas, L, Thingstad, TF (2002) Prokaryotic diversity: magnitude, dynamics, and controlling factors. Science 296: 1064-1066

67. Treves, DS, Xia, B, Zhou, J, Tiedje, JM (2003) A two-species test of the hypothesis that spatial isolation influences microbial diversity in soil. Microb Ecol 45: 20-28

68. van Belkum, A, Struelens, M, de Visser, A, Verbrugh, H, Tibayrenc, M (2001) Role of genomic typing in taxonomy, evolutionary genetics, and microbial epidemiology. Clin Microbiol Rev 14: 547-560

69. Vandamme, P, Pot, B, Gillis, M, de Vos, P, Kersters, K, Swings, J (1996) Polyphasic taxonomy, a consensus approach to bacterial systematics. Microbiol Rev 60: 407-438

70. Vinuesa, P, Silva, C, Werner, D, Martínez-Romero, E (2005) Population genetics and phylogenetic inference in bacterial molecular systematics: the roles of migration and recombination in Bradyrhizobium species cohesion and delineation. Mol Phylogenet Evol 34: 29-54

71. Ward, DM (1998) A natural species concept for prokaryotes. Curr Opin Microbiol 1: 271-277

72. Ward, DM, Weller, R, Bateson, MM (1990) 16S rRNA sequences reveal numerous uncultured microorganisms in a natural community. Nature 345: 63-65

73. Wayne, LG, Brenner, DJ, Colwell, RR, Grimont, PAD, Kandler, O, Krichevsky, L, Moore, LH, Moore, C, Murray, RGE, Stackebrandt, MP, Starr, MP, Trüper, HG (1987) Report of the ad hoc committee on reconciliation of approaches to bacterial systematics. Int J Syst Bacteriol 37: 463-464

74. Whitaker, RJ, Grogan, DW, Taylor, JT (2003) Geographic barriers isolate endemic populations of hyperthermophilic archaea. Science 301: 976-978

75. White, D, Phelps, T, Onstott, T (1998) What's up down there? Curr Opin Microbiol 1: 286-290

76. Wilson, M, Campbell, HL, Ji, P, Jones, JB, Cuppels, DA (2002) Biological control of bacterial speck of tomato under field conditions at several locations in north America. Phytopathology 92: $1284-1292$

77. Wu, L, Thompson, DK, Liu, X, Fields, MW, Bagwell, CE, Tiedje, JM, Zhou, JM (2004) Development and evaluation of microarray-based whole-genome hybridization for detection of microorganisms within the context of environmental applications. Environ Sci Technol 38: 6775-6782

78. Yu, Q, Alvarez, AM, Moore, PH, Zee, F, Kim, MS, de Silva, A, Hepperly, PR, Ming, R (2003) Molecular diversity of Ralstonia solanacearum isolated from ginger in Hawaii. Phytopathology 93: $1124-1130$

79. Zhou, JZ, Xia, BC, Treves, DS, Wu, LY, Marsh, TL, O’Neill, RV, Palumbo, AV, Tiedje, JM (2002) Spatial and resource factors influencing high microbial diversity in soil. Appl Environ Microbiol 68: 326-334 